\title{
On the Accuracy in the Derivation of Elemental Abundances in HII Galaxies
}

\author{
G. F. Hägele ${ }^{1}$, A. I. Díaz ${ }^{1}$, E. Pérez-Montero ${ }^{1}$, E. Terlevich ${ }^{2}$, \\ and R. Terlevich ${ }^{2}$ \\ ${ }^{1}$ Dpto. de Física Teórica, C-XI, Universidad Autónoma de Madrid, Cantoblanco, \\ 28049-Madrid, Spain \\ ${ }^{2}$ Instituto Nacional de Astrofísica, Óptica y Electrónica, Tonantzintla, Puebla, México
}

\begin{abstract}
We propose a methodology to perform a self-consistent analysis of the physical properties of the emitting gas of HII galaxies adequate to the data that can be obtained with the XXI century technology. This methodology requires the production and calibration of empirical relations between the different line temperatures that should superseed currently used ones based on very simple, and poorly tested, photo-ionization model sequences.

To reach these goals we have obtained simultaneous blue to far red longslit spectra with the William Herschel Telescope (WHT) in La Palma and the $3.5 \mathrm{~m}$ telescope at the Centro Astronómico Hispano Alemán (CAHA) of three (Hägele et al. 2006.MNRAS.372.293) and seven (Hägele et al. in preparation) compact HII galaxies respectively. The objects were selected from the Sloan Digital Sky Survey (SDSS) Data Release 3 (DR3) spectral catalog using the INAOE Virtual Observatory superserver.

For the observed objects we have measured at least four line temperatures: T([OIII]), T([SiII $]$ ), $\mathrm{T}([\mathrm{OII}])$ and $\mathrm{T}([\mathrm{SII}])$, and in three cases also $\mathrm{T}([\mathrm{NII}])$. The relation between $\mathrm{T}([\mathrm{SIII}])$ and $\mathrm{T}([\mathrm{OIII}])$ can be fitted with a unique empirical calibration. The actual fit to the data is : $\mathrm{T}([\mathrm{SIII}])=$ $(1.19 \pm 0.08) \mathrm{T}([\mathrm{OIII}])-(0.32 \pm 0.10)$. In contrast, for the relation between $\mathrm{T}([\mathrm{OII}])$ and $\mathrm{T}([\mathrm{OIII}])$ there is no single empirical calibration and it is not possible to give an estimate of the error introduced by the application of this procedure.

We have also measured the Balmer continuum temperature $\mathrm{T}(\mathrm{Bac})$ for the three WHT objects and derived the temperature fluctuations as defined by Peimbert (1967). Only for one of these objects, the temperature fluctuation is significant and could lead to higher oxygen abundances by about 0.20 dex (Hägele et al. 2006.MNRAS.372.293).

The measurements of several different line temperatures and a careful and realistic treatment of the observational errors yield total oxygen abundances with accuracies between 5 and $10 \%$. The fractional error is as low as $2 \%$ for the ionic $\mathrm{O}^{2+} / \mathrm{H}^{+}$ratio and increases to $15 \%$ for the $\mathrm{O}^{+} / \mathrm{H}^{+}$ratio. These accuracies are expected to improve as better calibrations based on more precise measurements, both on electron temperatures and densities, are produced. The accuracies are lower in the case of the abundances of sulphur (of the order of $20 \%$ for $\mathrm{S}^{+}$and $12 \%$ for $\mathrm{S}^{2+}$ ), the error increases further (up to $30 \%$ ) for the total abundance of sulphur due to the uncertainties in the ionization correction fractions (ICF).

This is in contrast with the small errors quoted for line temperatures other than $\mathrm{T}([\mathrm{OIII}])$ in the literature, in part due to the commonly adopted methodology of deriving them from the measured $\mathrm{T}([\mathrm{OIII}])$ through theoretical relations. These relations are obtained from photoionization models and no uncertainty is attached to them. If this methodology were to be adopted, the theoretical relations should be adequately tested and empirical relations between the relevant line temperatures should be obtained in order to quantify the corresponding model uncertainties.
\end{abstract}

Keywords. methods: data analysis, ISM: abundances, galaxies: abundances, galaxies: fundamental parameters, galaxies: starburst 